山्ञ FRANÇAISE

$\supset \mathrm{DE}$

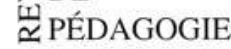

\section{Revue française de pédagogie}

Recherches en éducation

157 | octobre-décembre 2006

PISA : analyses secondaires, questions et débats

théoriques et méthodologiques

\title{
Procédés discursifs et rôles sociaux d'élèves en groupes de discussion sur une controverse socio- scientifique
}

Students' discursive processes and social roles in discussion groups discussing a socio-scientific controversy

Procedimientos discursivos y papeles sociales de alumnos en grupos de discusión sobre una controversia socio-científica

Diskursive Vorgänge und soziale Rollen der Schüler in Diskussionsgruppen über eine gesellschaftlich-wissenschaftliche Auseinandersetzung

\section{Virginie Albe}

\section{OpenEdition \\ Journals}

Édition électronique

URL : http://journals.openedition.org/rfp/604

DOI : $10.4000 /$ rfp. 604

ISSN : 2105-2913

Éditeur

ENS Éditions

Édition imprimée

Date de publication : 1 décembre 2006

Pagination : 103-118

ISBN : 978-2-7342-1075-7

ISSN : 0556-7807

Référence électronique

Virginie Albe, «Procédés discursifs et rôles sociaux d'élèves en groupes de discussion sur une controverse socio-scientifique », Revue française de pédagogie [En ligne], 157 | octobre-décembre 2006, mis en ligne le 01 décembre 2010, consulté le 02 mai 2019. URL : http://journals.openedition.org/ rfp/604; DOI : 10.4000/rfp.604 


\section{Varia}

\section{Procédés discursifs et rôles sociaux d'élèves en groupes de discussion sur une controverse socio-scientifique}

Virginie Albe

L'étude de questions socio-scientifiques en classe a été proposée afin de contribuer à la démocratisation des sciences. À cette fin, des situations favorisant les pratiques discursives entre élèves ont été développées. Nous avons étudié comment des élèves de première appréhendent une controverse socio-scientifique actuelle en groupes de discussion. Dans une approche micro-ethnographique, nous avons identifié différents procédés de discussion collective dans les groupes. Les résultats montrent que des facteurs épistémologiques, contextuels, et sociaux influencent l'élaboration de connaissances dans ces situations.

Descripteurs (TEE) : apprentissage, argumentation, conditions d'apprentissage, éducation scientifique, travail par équipe.

\section{INTRODUCTION}

L'enseignement scientifique est actuellement considéré en crise par de nombreux acteurs: politiques, enseignants, scientifiques, journalistes, etc. Face à ce que certains nomment la " désaffection des jeunes pour les sciences ", beaucoup s'interrogent sur les causes de cette "crise " et tentent de formuler des propositions (Royal Society [GB], 1985 ; Union européenne, 1995 ; OECD, 1998). L'adéquation de la formation des jeunes à l'emploi, les évolutions sociales, la mondialisation de l'économie, la complexité du monde, le déficit du sens dans les sociétés technocratiques, l'image des sciences et des scientifiques dans la société, la place et le rôle des médias sont questionnés (1).

Le système de formation et les pratiques pédagogiques sont également interrogés. Dans le champ de l'éducation aux sciences, les phénomènes d'enseignement et d'apprentissage font l'objet de recherche depuis des décennies. L'étude des pratiques effectives a notamment permis d'établir le constat d'un enseignement des sciences essentiellement selon une perspective positiviste (Aikenhead, 2003 ; Driver et al. 2000 ; Fourez 2002). Pour Fleming (1986), la science scolaire s'intéresse peu à la science. Elle traite de ses produits, de connaissances factuelles et les élèves la perçoivent comme une accumulation de faits. Dans 
ce contexte, de nombreux auteurs en appellent au changement (Désautels et al., 1993 ; Hodson, 2003 ; Millar \& Osborne, 1998; Solomon \& Aikenhead, 1994), soulignant qu'il est nécessaire de reconsidérer l'éducation scientifique, couramment vue comme une formation introductive en sciences qui se concentre sur le développement de compétences méthodologiques pour une formation sur les sciences.

De nouveaux programmes ont ainsi été mis en place dans divers pays dans le but d'une alphabétisation scientifique pour tous. Si ce but semble partagé par tous, les considérations sur ce qu'une personne alphabétisée doit être en mesure de réaliser sont très disparates.

Selon une orientation pragmatique, certains considèrent - puisque les sociétés sont de plus en plus influencées par les idées et les produits des sciences, et surtout des technologies - que tous les futurs citoyens seront mieux en mesure d'affronter les problèmes s'ils ont une base de connaissances scientifiques. II s'agit par exemple de développer une confiance rationnelle dans les experts lorsque l'on se trouve confronté à des questions incertaines et controversées (Shamos, 1995).

Pour d'autres, il s'agit d'utiliser les connaissances scientifiques dans un but de démocratisation. Dans cette orientation, on considère qu'une formation scientifique de qualité à l'école permet aux citoyens de participer significativement aux décisions que les sociétés doivent maintenant prendre à l'égard de problèmes socio-scientifiques et socio-technologiques toujours plus complexes (production et consommation d'énergie, sécurité alimentaire par exemple).

Prenant en compte les préoccupations actuelles en Amérique du Nord, Hodson (2003) propose par exemple de construire des curricula dans une visée d'action socio-politique autour de sept thèmes: la santé humaine, l'alimentation et l'agriculture, les ressources naturelles (eau, terre, minéraux), les ressources et la consommation d'énergie, l'industrie, les technologies de l'information et les transports, la responsabilité éthique et sociale. Considérant que les liens entre l'éducation aux sciences, la mondialisation économique, l'augmentation de la production et l'expansion sans limites menacent les libertés individuelles, le bien-être des sociétés et le futur de la planète, Hodson souhaite que ce curriculum constitue un engagement à poursuivre la refondation des valeurs des sociétés occidentales. II souligne qu'une formation à la nature des sciences et aux pratiques scientifiques est un élément clé dans le dévelop- pement d'une alphabétisation scientifique critique favorisant une participation active dans nos sociétés démocratiques.

Dans la même perspective de démocratisation des sciences, Driver et al. (2000) proposent de favoriser en classe la formation socio-épistémologique des élèves et soulignent l'importance de mettre l'accent sur le rôle de l'argumentation dans l'élaboration des connaissances scientifiques. Ils considèrent que pour que des individus s'engagent dans des débats, il est nécessaire qu'ils connaissent la façon dont des arguments sont élaborés en science et qu'ils soient capables d'évaluer les utilisations possibles et les limites des savoirs experts pour appuyer des points de vue.

Leur approche s'inscrit dans l'esprit de l'épistémologie contemporaine et s'appuie sur les résultats de la sociologie des sciences considérant que les faits scientifiques sont élaborés au sein d'une communauté par des pratiques socio-discursives et la confrontation à la « récalcitrance » du réel.

Des questions telles que les méthodes de recherche (épidémiologie par exemple), les aspects institutionnels des sciences (sociologie) devraient ainsi faire l'objet d'enseignements et Driver et al. (2000) proposent que les élèves pratiquent eux-mêmes le débat et l'argumentation lors d'activités portant sur la science en train de se faire (situations historiques ou contemporaines) alors qu'aujourd'hui, la parole en classe leur est peu donnée.

Ainsi, viser la démocratisation des sciences à l'école nécessite de proposer des situations destinées à favoriser le développement d'une épistémologie renouvelée et le développement des capacités argumentatives des élèves.

Il est dans ce cadre nécessaire de recentrer les activités pédagogiques et de les articuler à des questions vives sur le plan social et significatives pour les élèves.

Une des voies proposées concerne l'introduction dans les programmes de questions socioscientifiques controversées (réchauffement du climat, modes de production d'énergie, clonage, etc.). Dans ces situations, une participation active des élèves est recommandée (France, 1999 \& 2000) (2). À l'appui de travaux qui soulignent que les apprentissages scientifiques et le langage sont inextricablement liés, il est alors proposé de mettre en place des situations où les interactions langagières entre élèves sont favorisées (travaux en petits groupes, débats, cours dialogués, exposés, etc.). Au lieu d'être considérées comme 
des alternatives non problématiques aux modèles d'enseignement transmissifs, ces activités font l'objet de recherches depuis plusieurs années (Barnes \& Todd, 1977 ; Kelly \& Crawford, 1996 ; Gilly, 1988 ; Schubauer-Leoni \& Perret-Clermont, 1980). En particulier, des travaux en didactique des sciences et des langues ont porté sur l'impact des interactions entre élèves dans la construction de savoirs scolaires en sciences (Amigues, 1988 ; Fillon \& Peterfalvi, 2004 ; Garcia-Debanc, 1995 ; Jaubert \& Rebière, 2001).

Dans cet article, nous présentons une étude de l'impact de situations interactives entre élèves sur leur appréhension d'une controverse socio-scientifique. En particulier, nous avons tenté de cerner comment les interactions sociales, articulées aux confrontations avec des discours scientifiques controversés, interviennent dans les productions discursives des élèves lors de petits groupes de discussion.

\section{LE DISCOURS EN CLASSE DE SCIENCES, L'ÉTUDE DE QUESTIONS SCIENTIFIQUES SOCIALEMENT VIVES}

Des études ont montré l'importance des échanges oraux entre élèves pour leur permettre de développer leur compréhension conceptuelle (Barnes, 1977 ; Barnes \& Todd, 1977 ; Lemke, 1990 ; Sutton, 1995). Ceci met en avant les « relations étroites qui apparaissent, de plus en plus clairement, entre savoirs et productions langagières " (Jacobi \& Peterfalvi, 2004 , p. 4). Garcia-Debanc (1995, p. 102) souligne que "les interactions verbales apparaissent comme une composante essentielle de la construction des apprentissages dans une démarche scientifique ». De même Jaubert et Rebière (2001) considèrent qu'il ne peut y avoir construction de savoirs sans la construction simultanée des discours qui permettent de dire ces savoirs.

L'effet des pratiques de travail en petits groupes sur les apprentissages a ainsi fait l'objet de recherches dans le domaine de l'éducation aux sciences. Des auteurs ont montré que les relations entre les élèves, leur statut académique, leur genre influencent et structurent les interactions dans les groupes (Bianchini, 1997 ; Goffard \& Goffard, 2003 ; Moje \& Shepardson, 1998). Les rôles sociaux adoptés par les élèves au cours des discussions de groupe ont également été étudiés et cela a permis de montrer un effet de «leader » sur les connaissances élaborées au sein des groupes (Richmond \& Striley, 1996). Par ailleurs, l'analyse de l'influence des contextes d'enseignement a permis de montrer que les élèves ont souvent des difficultés à travailler collectivement (Alexopoulou \& Driver, 1996 ; Bianchini, 1997 ; Kittleson \& Southerland, 2004). D'autres recherches ont porté sur les procédés d'élaboration collective des savoirs entre élèves en groupes de discussion (Kelly \& Crawford, 1997). Différents modes de coopération ont été identifiés (Gilly, 1988 ; Goffard \& Goffard, 2003) et les dynamiques des échanges dans les groupes de discussion ont fait l'objet d'analyses (Schneeberger \& Ponce, 2003). Si le rôle important des situations de collaboration entre pairs dans la marche vers l'élaboration des connaissances semble faire l'unanimité, la plupart des auteurs signalent que ce n'est pas nécessairement un processus productif.

Fillon et Peterfalvi (2004) s'interrogent par exemple sur l'impact des discussions de groupe sur les apprentissages des élèves. En référence à Meirieu qui " dès 1984, proposait une interrogation critique sur l'effet des pratiques de travail en groupe sur les apprentissages, ils indiquent que l'engagement cognitif des élèves peut s'avérer très inégal selon leur position dans le groupe ou par rapport à l'enseignant" (Ibid., p 176). Pour Dawes (2004) si les apprentissages scientifiques et le langage sont inextricablement liés, les groupes de discussion ne conduisent pas nécessairement à une collaboration des élèves en vue de construire des connaissances dans la mesure où la demande sociale de la situation est souvent trop forte.

Par ailleurs, les travaux qui ont porté sur l'étude par les élèves de controverses socio-scientifiques montrent qu'il s'agit de phénomènes complexes (Bader, 2003 ; Sadler, Chambers \& Zeidler, 2004 ; Schweizer \& Kelly, 2001 à propos du réchauffement climatique ; Albe, 2005 sur les technologies de la téléphonie mobile; Simonneaux, 2001 \& 2003 sur les biotechnologies). Plusieurs tendances peuvent toutefois être identifiées: l'influence significative de l'expérience personnelle des élèves et de leurs émotions, la prise en compte de considérations sociales et l'importance de considérations épistémologiques, et, dans certains cas, la primauté de considérations morales (Sadler \& Zeidler, 2005). La mobilisation de connaissances scientifiques et technologiques par les élèves dans l'étude de ces controverses a également été questionnée.

L'expérience personnelle apparaît comme un moyen pour interpréter et problématiser la controverse, une référence pour argumenter et justifier une position : étude par des lycéens de questions environnemen- 
tales (Patronis et al., 1999), liées à la thérapie génique (Sadler \& Zeidler, 2004) et à l'énergie nucléaire (Fleming, 1986). Pour certains auteurs, les émotions ont un effet facilitateur pour engager les élèves dans l'étude de controverses socio-scientifiques (Sadler et al., 2004). L'empathie ou la sollicitude peuvent en particulier permettre de prendre en considération les points de vue d'autres individus ou de cerner l'impact individuel et social des décisions prises. Ainsi, dans la recherche de Sadler \& Zeidler (2005) les élèves se sont identifiés aux personnages mis en scène dans les scénarios socio-scientifiques proposés sur le clonage et ont adopté une perspective plurielle pour appréhender des points de vue opposés et controversés (Sadler \& Zeidler, 2005).

Par ailleurs, des recherches ont montré qu'un engagement émotionnel important accompagné de la capacité de réguler et contrôler cet engagement par des mécanismes communicationnels et sociaux ont conduit à développer les points de vue empathiques, les comportements en faveur d'action sociale et les comportements visant à faire face aux problèmes de façon constructive (Eisenberg, 2000).

Si ces résultats plaident en faveur de la prise en compte du rôle des émotions dans l'éducation aux sciences alors que traditionnellement seul un raisonnement rationaliste est [positivement] considéré [ou uniquement pris en considération], il nous semble que dans ce domaine également une attitude de prudence soit nécessaire.

De même que mettre des élèves en petits groupes ne conduit pas nécessairement à une co-construction de connaissances, proposer aux élèves des situations portant sur des controverses socio-scientifiques ayant une résonance émotionnelle forte n'assure pas leur engagement dans leur étude. Si des questions telles que l'usage de téléphones cellulaires, la production et la consommation d'OGM, le réchauffement climatique, etc. permettent de rejoindre des préoccupations personnelles des élèves, ce qui est peu le cas dans les cours de sciences traditionnels, il se peut également qu'une dimension affective forte dans ces controverses ait un effet inhibiteur, des élèves pouvant être amenés à taire leurs points de vue dans des discussions qui les impliqueraient trop personnellement. Par ailleurs, la confrontation des élèves à des controverses où la science est incertaine et ne peut fournir de certitudes, de faits incontestables qui font autorité comme lors des enseignements traditionnels peut être déstabilisante pour les élèves et les enseignants (Hind, Leach \& Ryder, 2001) et générer anxiété, vulnérabilité et insécurité.
Dans la mesure où les controverses socioscientifiques peuvent avoir une dimension affective forte, pour Dewhurst (1992) adopter un autre point de vue que le sien peut aider à mieux comprendre mais il signale que ce n'est pas un processus évident. II nous semble également qu'un recours aux émotions pour engager des élèves dans l'étude de controverses pourrait conduire à une polarisation des points de vue, ce qui peut être un obstacle à la compréhension de questions complexes.

Pour d'autres auteurs, la prise en compte par les élèves de considérations sociales jouent un rôle majeur dans leur appréhension de controverses socio-scientifiques. Fleming (1986) a montré que les étudiants raisonnent principalement la question d'accepter un emploi dans une centrale nucléaire dans le domaine social en évaluant notamment la possibilité d'effets sur d'autres individus ou dans le domaine personnel en recherchant un intérêt individuel sur le plan économique. Les élèves utilisent peu de connaissances scientifiques et ne considèrent pas les connaissances scientifiques scolaires comme pertinentes. Des étudiants précisent par exemple qu'il leur faudrait plus que la science scolaire sur les radiations comme par exemple connaître les effets sur la santé des personnes exposées. Tous les élèves interrogés seraient prêts à s'engager dans des études en classe sur ces aspects scientifiques.

Si les connaissances scientifiques sont réduites à l'emploi de quelques terminologies face à ces questions socio-scientifiques, en revanche, les connaissances sur les scientifiques et la science comme une institution constituent les connaissances les plus couramment utilisées par les étudiants (Fleming, 1986). La science pour les étudiants est une accumulation de faits (c'est ce qu'ils connaissent de la science scolaire), la technologie utilise ces faits afin de conduire au progrès pour faire avancer "notre civilisation". Ainsi, ils considèrent qu'avec plus de faits, on résoudrait la question socio-scientifique.

Par ailleurs, pour Yang et Anderson (2003), les considérations sociales sont le plus souvent articulées à des considérations scientifiques. De même pour Sadler et al. (2004), les étudiants considéraient les données scientifiques associées à différentes positions ainsi que les conséquences sociales de ces positions.

Peu de travaux se sont centrés sur les pratiques discursives des élèves lors de groupes de discussion portant sur des controverses socio-scientifiques. 
Leach \& Lewis (2002) ont décrit les manières de parler au sein de groupes discutant du diagnostic prénatal de la fibrose cystique, en utilisant les catégories de Mercer (1996). Ils ont observé quelques groupes, composés de filles, avec un style de discours " d'exploration ». Dans les autres groupes, les élèves répètent leurs avis personnels, ne tentent pas de les justifier ou d'argumenter pour s'opposer aux affirmations opposées.

Dans la mesure où les questions soulevées par ces controverses sont porteuses d'affect, où les connaissances scientifiques élaborées dans la cité savante sont incertaines et controversées, et par conséquent une maîtrise des contenus académiques de la science scolaire n'offre que peu de recours, comment les élèves appréhendent la controverse dans le cas de groupes de discussions? Quels procédés discursifs élaborent-ils collectivement ? Quelle organisation sociale développent-ils dans la discussion? Quels sont les éléments qui influencent les connaissances élaborées dans les groupes? Telles sont les questions que nous avons étudiées dans la présente recherche.

\section{ANCRAGES THÉORIQUES}

Dans une approche située de la cognition, il s'agit "d'envisager les processus cognitifs et l'activité comme indissociables d'une situation, dont les éléments physiques, artefactuels autant que sociaux, offrent des ressources signifiantes pour l'action des sujets " (Grison, 2004).

La cognition de l'être humain y est vue comme incarnée et historiquement construite. Les connaissances sont un résultat de l'activité et des situations dans lesquelles elles ont été produites. La cognition située met également l'accent sur le caractère social de la construction des connaissances. L'apprentissage est réalisé par des interactions sociales collaboratives (Resnick, 1988). Autrement dit, dans l'activité, le sens est socialement construit à travers les négociations entre acteurs (Brown, Collins \& Duguid, 1989). Cette perspective de la cognition défend une posture anti-mentaliste (Coulter, 1979) et rejoint la sociologie interactionniste et l'ethnométhodologie (Garfinkel).

L'interactionnisme symbolique met également l'accent sur la dynamique co-constructive du sens considérant que la signification donnée à une situation se crée pendant l'interaction. La perspective prag- matique de l'interactionnisme rejoint ainsi les approches constructivistes de la cognition. Cette approche met aussi en avant la complexité des rôles sociaux et le caractère dynamique et négocié de l'interaction sociale. Les rôles sociaux sont le résultat de processus complexes où chaque acteur participe à la représentation de la situation dans laquelle il est engagé, où le déroulement de l'interaction n'est jamais figé, mais sans cesse ré-adapté, réinvesti, négocié et donc, bien plus le fait de conflits, de négociations, de divergences d'intérêts, de tensions affectives que la conséquence de règles strictes et fonctionnelles - souvent ignorées d'ailleurs - par les acteurs auxquels elles sont censées s'appliquer (Strauss, 1992).

Quant à l'ethnométhodologie qui s'attache à comprendre comment le langage est imbriqué dans l'organisation des activités sociales, elle ne recourt pas à des explications intentionnelles ou psychologiques pour expliquer les actions parce ce qu'elle les considère d'abord comme des constructions discursives.

Ainsi, les points de vue sur la cognition, les interactions sociales et le langage se rejoignent pour considérer que les connaissances sont co-construites à travers les interactions interpersonnelles en situation grâce à une réalité intersubjective reposant sur des symboles langagiers partagés.

Afin de cerner les procédés discursifs collectivement élaborés par les élèves en groupes de discussion lors de l'étude d'une question scientifique socialement vive, nous avons opté pour une approche focalisée sur les pratiques des locuteurs qui mobilisent des ressources en situation, et ainsi tient compte des orientations des participants dans l'action. Mettant l'accent sur la dimension située et interactive des pratiques langagières, nous aurons recours à la notion d'objet de discours. Comme le souligne Mondada (2005, p. 23), "les objets de discours sont interactivement proposés, repris, ratifiés, transformés, rejetés par les interlocuteurs. Ces objets sont ainsi constitués au fil des activités communicationnelles ; lls construisent une "version publique du monde ». Celle-ci est élaborée en adéquation avec les relations intersubjectives et sociales des participants et avec le contexte social dans lequel elle est formulée. ”

Par ailleurs, nous nous appuyons sur l'interactionnisme symbolique pour étudier l'organisation sociale développée par les élèves dans la discussion, considérant que l'ordre social au sein des groupes de discussions est un ordre négocié. Pour les interactionnistes symboliques, les structures se construisent 
pendant les interactions entre acteurs et sont le résultat de ce processus. Ainsi, dans cette étude, nous considérons que les rôles sociaux sont le fruit de l'activité des membres des groupes de discussion. Ces rôles sont structurés par les élèves à travers les processus d'interaction. Ils se construisent, évoluent et se négocient dans les interactions. Ces rôles sociaux sont également structurants des discussions au sein des petits groupes.

\section{CONSIDÉRATIONS MÉTHODOLOGIQUES}

« Les téléphones cellulaires sont-ils dangereux pour la santé ? » (3) est une situation-débat (Albe \& Simonneaux, 2003) inspirée d'un module de formation développé par un groupe de chercheurs et d'enseignants pour favoriser la formation épistémologique des élèves (Hind et al., 2001).

Nous avons conduit cette étude de la question controversée de la dangerosité des téléphones cellulaires avec une classe de douze élèves de série technologique de l'enseignement agricole (Sciences et technologies de l'agronomie et de l'environnement) pendant une séance ordinaire d'environ deux heures. II s'agit de préparer et de réaliser un jeu de rôle: les élèves jouent le rôle d'avocats dans le cas d'un procès où un employé poursuit son employeur pour son mauvais état de santé, qu'il estime dû à l'usage du téléphone portable et en raison duquel il a dû quitter son travail. Les élèves se répartissent en deux groupes: les avocats de la partie civile et de la défense. Le groupe $A$ défend la victime et la thèse que les téléphones portables sont dangereux pour la santé ; le groupe $B$ défend l'employeur et la thèse que les téléphones portables ne sont pas dangereux pour la santé.

Un dossier est distribué aux élèves. II est constitué de sept extraits de recherche actuelles portant sur l'apparition de maladies sur des animaux, des enquêtes épidémiologiques, des tests sur la mémoire. Chaque groupe étudie les documents et prépare des arguments pour le jeu de rôle.

Toute la leçon a fait l'objet d'enregistrements audio et vidéo. La totalité des échanges ont été retranscrits (4).

Considérant que le langage est une activité sociale qui permet l'élaboration du sens et que structuration de la pensée et échanges langagiers sont liés (Billig, 1996), nous nous sommes centrée sur les pratiques discursives des élèves et leur construction de savoirs plutôt que sur les caractéristiques linguistiques du discours lui-même (Edwards \& Mercer, 1987). Considérant les connaissances élaborées par les élèves dans cette activité comme locales, contingentes et dépendantes des groupes sociaux, nous avons analysé les pratiques discursives au sein des groupes d'élèves sans séparer la cognition du social, comme le suggèrent Kelly et Crawford (1997). Pour cela, nous avons effectué une analyse de discours d'inspiration ethnométhodologique car comme l'indique Mondada (2005, p. 84) « cette perspective permet d'observer, à la fois, le fonctionnement d'un collectif et la production collective des savoirs, en veillant à ne pas les séparer l'un de l'autre ». Ainsi, nous nous sommes focalisée sur les procédés d'élaboration collective des savoirs, autrement dit les procédés par lesquels des objets de discours introduits, identifiés et reconnus comme tels par les participants à la discussion sont développés (Mondada, 2005). Nous analysons l'ensemble des échanges au sein des groupes dans la mesure où les interventions des élèves dépendent du contexte de l'ensemble de la discussion (Pontecorvo, 1993). Comme le souligne Jimenez-Aleixandre (2003), dans le modèle de la cognition située, on considère qu'apprendre les sciences c'est être apprenti de pratiques discursives de la communauté scientifique. La co-construction de connaissances par les élèves a lieu lors de dialogues interactifs. Les arguments construits en collaboration entre deux ou plusieurs personnes tiennent compte des arguments des autres, pour les appuyer ou pour s'opposer à eux. II faut ainsi procéder à une analyse de l'ensemble des discours produits, dans la mesure où une proposition d'un locuteur tient compte des échanges précédents. Pour cela, nous suivons pas à pas les échanges verbaux des élèves.

Pour ce faire, les outils d'analyse développés n'étaient pas pré-déterminés à la collecte de données mais ont été construits lors de multiples confrontations avec les données recueillies.

Dans un premier temps, nous repérons dans l'ensemble du corpus les passages où les échanges entre les participants à la discussion sont centrés sur un objet de discours. Les élèves élaborent collectivement ces objets, ils ne pré-existent pas à leur discussion dans l'interaction mais peuvent par exemple faire intervenir des éléments de savoirs fournis dans les extraits de recherche à étudier, qui seront alors négociés et retenus comme plausibles par les élèves.

Nous procédons ensuite à une analyse microscopique des épisodes précédemment identifiés. 
Une tendance actuelle majeure consiste à généraliser toute activité discursive à l'argumentation. Pour Plantin (1996), "du point de vue du dialogue, peut être considéré comme argumentatif tout discours produit dans un contexte de débat orienté par une question ". Cette définition nous semble particulièrement adaptée à notre situation où les élèves se trouvent confrontés à une controverse socio-scientifique. Face à des discours scientifiques contradictoires, les élèves développent des pratiques argumentatives. Pour décrire ces pratiques, nous nous sommes inspirée de travaux de didacticiens portant sur l'argumentation en classe de sciences (Jiménez-Aleixandre et al., 2000 ; Simonneaux 2003 ; Weisser, Masclet \& Remigny, 2003). Le modèle de Toulmin a été considéré comme peu pertinent pour analyser l'argumentation dans des débats sur des questions scientifiques socialement vives (Simonneaux, 2003) et insuffisant pour rendre compte de ce qui s'échange dans la dynamique des interactions verbales (Fillon \& Peterfalvi, 2005).

Afin de saisir les pratiques argumentatives des élèves et leur dimension sociale, l'analyse des tours de parole nous permet de repérer la nature et le contenu des interventions des élèves. II peut s'agir de questions, propositions avec ou sans justifications, oppositions, réfutations, d'expressions d'accord ou de désaccord, de répétitions, de reformulations (Simonneaux 2003 ; Weisser, Masclet \& Remigny, 2003). Les élèves peuvent mettre en discussion des éléments issus des extraits de recherche, des considérations stratégiques, sociales, des connaissances scientifiques, techniques, profanes, des points de vue sur les sciences, sur le travail du groupe, etc. Nous identifions également les élèves qui sont à l'initiative et à la clôture des épisodes (Jiménez-Aleixandre et al., 2000), ceux qui éventuellement gèrent les prises de parole dans le groupe, les transitions entre les différents épisodes de l'activité, qui évaluent, soutiennent ou contestent les propositions des autres, font usage d'autorité, soulèvent des critiques, etc., afin de caractériser les rôles sociaux développés dans les interactions entre élèves.

\section{RÉSULTATS}

Les élèves du groupe A mettent en œuvre plusieurs procédés collaboratifs à partir des extraits de recherche, de connaissances quotidiennes et de considérations socialement partagées ou «truismes culturels "
(Billig, 1987). L'étude de la trajectoire des objets de discours montre plusieurs reprises des objets de discours, pouvant conduire à une argumentation collaborative. Dans certains cas, les élèves ont articulé leurs désaccords lors de procédés discursifs complexes.

Dans l'extrait suivant par exemple, qui a lieu au début de la discussion du groupe, il s'agit pour les élèves de statuer sur le fait que le lien entre les résultats obtenus sur des tissus animaux et les résultats chez les humains soit valide ou non. Ceci fait l'objet de confrontations contradictoires.

7. Arnaud ${ }^{\star}$ : De toute façon on a tout intérêt à dire qu'il y a un lien.

[Interruption par l'enseignante]

8. Sébastien: Mais justement, le lien, il faut le démontrer.

9. Arnaud: Hé bien, tu mets que ce sont des cellules animales, et que nous aussi on est constitués de cellules animales. Pourquoi ça marcherait chez l'un et pas chez l'autre?

10. Arnaud: On peut leur dire: "Non, non, il n'y a pas de problème, c'est pas dangereux. "

[interruption par l'enseignante pour préciser les consignes de l'activité]

15. Sébastien : Bon, [Arnaud], pour le premier, là. Moi je te dis que ce n'est pas cohérent parce que nous on est des mammifères et eux c'est des cellules animales.

16. Katia : Oui on est des mammifères et eux c'est des cellules animales.

17. Arnaud: Oui, mais c'est quand même le règne animal.

18. Sébastien: Oui, mais il est grand, le règne animal.

19. Arnaud: Tu ne vas pas leur dire aux autres: "Oui, de toute façon vous avez raison, le portable..."

20. Sébastien : On pourra dire oui, autre part, il y en a plein...

21. Arnaud : II faut dire oui partout.

22. William : Comme ça on est sûrs.

23. Arnaud: Eux ils vont dire non partout, alors...

24. Sébastien: Bon, donnez votre opinion, là, qu'on soit plusieurs et après on vote.

Arnaud fait une proposition stratégique, Sébastien exprime son désaccord et argumente. Katia acquiesce et par ce procédé collaboratif soutient l'argument énoncé par Sébastien. William appuie les propositions stratégiques de Arnaud, en désaccord avec Sébastien. Sébastien propose de voter et organise le recueil des points de vue des autres dans

\footnotetext{
* Nous utilisons des pseudonymes pour les prénoms des élèves.
} 
les échanges suivants, adoptant en cela un rôle de leader. II apparaît que les désaccords ont déstabilisé l'objet de discours jusqu'à sa fragmentation.

La discussion porte sur une question de nature ontologique relative au statut de l'homme et les élèves montrent des difficultés à élaborer collectivement un point de vue. Hill (1989) cité par Larochelle (2002) a d'ailleurs montré la difficulté pour les élèves de placer l'homme du côté de l'animal dans des catégorisations. Mais il apparaît également dans cet extrait que des considérations stratégiques orientent les discussions et façonnent les objets de discours.

Plus loin dans les échanges du groupe, à l'occasion d'une discussion sur les extraits à retenir pour justifier leur thèse, les élèves reviennent sur la question du lien entre les résultats obtenus sur des tissus animaux et les effets chez les humains.

Sébastien propose que l'extrait de recherche $n^{\circ} 2$ soit utilisé. William exprime son accord et le renforce en précisant la conclusion de cette recherche. Puis Sébastien change d'avis et suggère d'utiliser l'extrait de recherche $n^{\circ} 1$. William exprime son désaccord et le justifie en référence à un objet de discours élaboré au début de l'activité : la validité du lien entre résultats obtenus sur des animaux et effets sur l'homme est incertaine. II apparaît ici que les élèves tendent à exclure les résultats qui ne peuvent fournir de certitude, ce qui ne semble pas sans lien avec une certaine conception des sciences comme établissant des vérités, des preuves, des faits certains.

514. Sébastien : Bon, moi, j'ai mis la 2.

515. William: La 2 aussi, réceptive au changement, oui, oui, ça provoque des changements pour la mémoire.

516. Sébastien: Ha non, on pourrait utiliser la 1, je pense plutôt. Comme ça on peut dire...

517. William: Mais non, mais non, parce que c'est d'un lien possible. Alors là, on n'est vraiment pas sûrs.

518. Arnaud: Là, c'est les nématodes?

519. Sébastien: On peut dire que si ça agit sur les vers, alors ça a peut-être une incidence sur nous.

520. Arnaud: Voilà...C'est quand même avant tout des animaux enfin des...

521. Sébastien : Oui, c'est vivant, c'est...

Au tour 519, Sébastien propose un argument que Arnaud évalue au tour suivant puis complète. Sa formulation permet l'établissement d'un accord. En référence aux discussions précédentes (tours de parole 15 à 18), il place "vers » et «être humains " dans une même catégorie, "les animaux", puis nuance sa formulation avec "enfin », ce qui permet à Sébastien d'affirmer son accord et de compléter l'énoncé en train de se faire en reformulant la catégorie avec le terme "vivant ». On constate lors de cet épisode que l'élaboration collective a lieu entre deux élèves du groupe, un autre pouvant être considéré comme une aide à la discussion, les autres (les filles) n'y participant pas.

Dans l'extrait qui suit, les désaccords ne parviennent pas à être dépassés par la discussion.

194. Arnaud: Oui, mais tu peux dire qu'en dix-huit mois tu ne peux pas développer un cancer.

195. Sébastien : Oui, mais tu peux quand même avoir une tumeur qui se forme. Si ! elle grossit au fur et à mesure, la tumeur.

196. Arnaud: Oui mais ...

197. Agnès: Oui ça arrive vite aussi

[Interruption: changement de face de la cassette audio]

202. Arnaud: Moi je réponds que la durée est trop courte.

203. Agnès : Oui, oui

204. Katia: Mais non, parce que, là, tu vas dans leur sens à eux en disant ça.

205. Arnaud: Mais non!

206. William: mais non au contraire, si on l'avait laissé plus longtemps, peut-être qu'il y aurait eu des risques.

207. Arnaud: Plus tu exposes plus tu as de risques.

208. Katia : Ah oui OK

209. Sébastien : Et c'est où qu'ils... ? Ha oui, vingt minutes, quand même vingt minutes c'est à peu prés le temps qu'on... Tu ne passes même pas vingt minutes au téléphone par jour.

210. Arnaud : Enfin.

211. Sébastien: Hein, vingt minutes?

212. Arnaud: Certains, je suis certain qu'ils y passent...

213. Sébastien : tu as un forfait de combien ?

214. Arnaud: Pas forcément moi !

215. Agnès : c'est vrai, il y en a ils passent beaucoup de temps

216. Sébastien: En moyenne, en moyenne, on ne passe pas vingt minutes, non, c'est vingt heures, c'est vingt heures par jour, ouais, tu ne passes pas vingt heures par jour au téléphone.

217. William: Ça n'existe même pas les forfaits de vingt heures pour un mois alors!!

218. Sébastien : Alors sur dix-huit mois je pense que l'expérience, elle est valable.

219. William : Alors, ça ne veut rien dire.

220. Sébastien : vingt heures, ça veut dire que quatre heures de repos.

221. William : [inaudible] ...ton portable pendant cinq ans. 
222. Arnaud: Peut-être qu'en vingt heures, dix-huit mois c'est quand même trop court.

223. Agnès : oui, c'est vrai, c'est court

224. Sébastien : attends, c'est court?

225. Arnaud: C'est peut-être beaucoup par jour mais...

$[\ldots]$

229. Arnaud: C'est trop court, un an et demi, tu ne peux pas développer un cancer en un an et demi quand même.

230. Sébastien : Ha si, ha, ça dépend. Non, non, non, moi je te dis qu'elle est valable. Vingt heures quand même, vingt heures! Tu téléphones, allez, on va dire tu téléphones cinq minutes par jour

231. Arnaud: je m'en fous

232. Sébastien : allez, un peu plus, allez on va dire un quart d'heure, donc vingt heures...

233. Arnaud: mais je m'en fous de la quantité, moi. Moi je te dis: c'est le temps, ceux qui développent les cancers, les humains, ils n'ont pas dix ans. Ils ont quel âge ? entre quarante et soixante ans.

234. Sébastien: De toute façon, le téléphone, ça fait combien de temps que ça existe, alors ? non, dix-huit mois je pense que c'est valable.

235. William : non, c'est pas assez

236. Sébastien : On va voter, on va voter, qui est-ce qui est de mon avis?

La discussion porte au début de cet extrait entre Arnaud et Sébastien sur la durée d'exposition des souris dans la recherche. On note les formulations "oui mais " qui initient chaque prise de parole et indiquent l'opposition entre les deux locuteurs. Leurs propos indiquent qu'ils se réfèrent à la carcinogenèse humaine, ce qui semble traduire une tendance à l'anthropomorphisme dans leurs justifications comme d'autres auteurs l'ont observé (Jimenez et al., 2000 ; Grace, 2005). Arnaud réaffirme son point de vue (202) et trouve le soutien d'Agnès dans une double affirmation puis l'opposition de Katia qui la justifie par des considérations stratégiques. Arnaud et William justifient la durée insuffisante avec des formulations collaboratives (reprise des formulations du locuteur précédent, complétées ou reformulées). Puis Sébastien fait émerger un nouvel objet de discours qui a pour effet de ne pas stabiliser l'argument précédent et de déplacer le désaccord entre Sébastien et Arnaud sur ce nouvel objet de discours.

On note lors de leurs échanges que la référence est l'expérience personnelle (durées des forfaits, des communications téléphoniques, de repos journalier). $\mathrm{Au}$ tour 222, Arnaud réaffirme son point de vue y intégrant l'objet de discours introduit par Sébastien.
Agnès soutient à nouveau cet argument dans un procédé de co-élaboration acquiesçante (Gilly, 1988) et Sébastien s'y oppose sous une forme interrogative ce qui conduit Arnaud à développer son point de vue. Arnaud et Sébastien s'opposent ensuite et on note la référence à la carcinogenèse humaine et à des connaissances quotidiennes, socialement partagées ou truismes culturels selon l'expression de Billig (1987). Sébastien s'oppose en utilisant la formulation " de toute façon " ce qui semble indiquer qu'il ne peut que se ranger à l'argument de Arnaud, son inscription dans une "évidence sociale» ne pouvant être discutée. Puis, se plaçant également sur le plan social, Sébastien questionne la durée d'utilisation de cette technologie pour s'opposer à l'argument de Arnaud. Enfin, il réaffirme son opposition sans la justifier. William s'oppose sur le même mode: il affirme son désaccord sans le justifier ou l'argumenter. Sébastien propose alors de voter et sollicite les autres, dans un rôle de leader. Les désaccords ont ici pour effet une déstabilisation de l'objet de discours allant jusqu'à sa fragmentation.

Par ailleurs, les élèves discutent à plusieurs reprises des résultats obtenus sur l'hippocampe de rats dans l'extrait de recherche $n^{\circ} 3$. Lors d'un premier épisode de discussion de cette question, il apparaît que la prise en compte du discours potentiel de l'autre groupe (portée par Nicolas) permet aux élèves de développer des justifications. On constate ensuite que par deux fois un questionnement prenant en compte les contre arguments que pourrait avancer l'autre groupe (soulevé par Katia) ne crée pas d'opportunité pour développer le discours. Dans un cas, deux autres élèves assumant respectivement les rôles de leader et d'aide, collaborent sur un autre objet de discours. Dans l'autre cas, un autre objet de discours est collectivement discuté par l'élève dans le rôle de «leader » et deux élèves assumant le rôle d'aide dont l'élève ayant soulevé le questionnement (Katia et William). L'extrait suivant constitue le dernier épisode de discussion sur cette question.

587. Arnaud: Je n'ai pas compris I'hippocampe, là,...

588. Sébastien : Les arguments, on va dire quoi ?

589. Arnaud: L'hippocampe est trop profondément enfoui dans le cerveau.

590. Katia: Non, mais ça, c'est ce qu'ils risquent de dire si on dit que ça atteint...

591. Arnaud: Oui, mais si ça a effet sur quelque chose qui est au milieu du cerveau, pourquoi ça ne pourrait pas... 
592. Katia : Oui, parce que si ça atteint une partie du cerveau, après, les cellules elles vont se développer et puis atteindre le...

593. Arnaud: Et puis, il n'y a pas de raison que ça attaque que juste le milieu du cerveau et pas le reste.

594. Katia: Oui et puis, après, ça ce propage partout.

595. Sébastien : Ouais.Ouais,

596. William : Ouais, ça va se propager.

597. Katia: Oui, c'est comme la tumeur au début c'est un petit truc, et puis, après...

598. William : Ça grandit.

599. Arnaud: Tu peux dire que c'est peut-être l'endroit le plus sensible du cerveau, I'hippocampe justement, mais que...

600. Katia: mais dire que c'est un endroit précis... C'est une raison à la con.. [inaudible]

601. Arnaud: Ça ne tient pas debout.

602. Katia: Oui, c'est débile. Bon allez c'est parti. Donc recherche 2.

La question de l'hippocampe est reprise par Arnaud au tour 587 . Katia répète alors que cela pourrait être un argument que leur opposerait l'autre groupe. Arnaud propose un contre-argument: des effets ayant été observés sur l'hippocampe qui serait profondément enfoui dans le cerveau, pourquoi d'autres zones du cerveau ne seraient pas atteintes? Les autres acquiescent et prolongent cette idée. Pour Katia «si ça atteint une partie du cerveau [...] après, ça ce propage partout» (594). William acquiesce en reprenant la formulation de Katia (596) et en la reformulant (598). Par ces procédés collaboratifs, les élèves ont réalisé un accord sur cet argument. Arnaud et Katia partagent ici la construction de l'argumentation. Les autres acquiescent par divers procédés (simple accord, accord et prolongement du discours).

Dans le groupe de discussion B, il apparaît que les élèves éprouvent des difficultés pour faire émerger des objets de discours collectifs et ne parviennent pas à articuler leurs désaccords dans des processus de discussion collective. Le plus souvent, ils mettent en œuvre des procédés d'acquiescement (un élève fait une proposition qui est acceptée). Les procédés collaboratifs ont le plus souvent lieu entre deux élèves plutôt que dans le groupe de discussion comme dans l'extrait suivant où les élèves établissent la non fiabilité d'une recherche à partir de considérations sur le mode d'établissement des connaissances par les chercheurs.
396. Cécile : Hé bien, ça, ils ne sont pas sûrs...

397. Nathan : Ils se basent sur des hypothèses, donc, encore une fois.

398. Cécile : qu'ils croient

399. Nathan: Donc les chercheurs se basent sur des hypothèses qui ne sont pas forcément justes.

À plusieurs reprises, les élèves expriment leurs points de vue individuels plutôt qu'ils ne font émerger un objet de discours collectif comme l'illustre l'extrait suivant.

30. Fabien : Qu'est-ce que c'est que ça?

31. Caroline : Là il y a marqué : " une recherche plus récente "

32. Fabien : On met oui la seconde aussi, alors. Non, je me trompe.

33. Nathan : pour le débat, après, il faut qu'on soit du même avis quand même.

34. Fabien : Tu n'as qu'à imposer ton opinion.

35. Caroline: Non.

36. Fabien: Je veux dire: il n'a qu'à parler, au lieu de faire l'idiot.

37. Nathan: Je ne veux pas faire l'idiot, mais vous êtes : toi tu mets une réponse et Caroline elle met une autre réponse.

38. Caroline : [Inaudible]

39. Max: de toute façon, Fabien, tu comprends jamais rien

40. Sandra: Je sais c'est fait exprès

41. Max : qu'est-ce qu'il y a ?

42. Caroline : C'est oui, oui, c'est non?

43. Fabien : et faudrait pas foutre trois jours pour faire un document hé parce que sinon ça va pas! Non, c'est oui, oui ou...

Apparaît [apparaissent ?] ici des désaccords entre les élèves et des attaques personnelles. Une intervention ironique et le recentrage sur l'activité ont pour effet de ne pas conduire à un conflit et finalement deux élèves se mettent d'accord sous la forme d'une co-élaboration acquiescante pour affirmer qu'ils ne peuvent pas déterminer si cette recherche est reproductible.

L'adoption des rôles d'avocats peut permettre aux élèves de collaborer et de parvenir à un accord collectif. Dans l'extrait qui suit par exemple, les élèves ont collectivement établi que la recherche de l'extrait $n^{\circ} 4$ n'est pas valide car il s'agit de souris génétiquement modifiées. 
105. Fabien : On a utilisé des souris qui ont été génétiquement modifiées pour augmenter leur...

106. Max: Elles ont été génétiquement modifiées : c'est pas bien.

107. Nathan: Non, mais, ils disent qu'elles ont été génétiquement modifiées.

108. Une fille: Oui.

109. Nathan: Ça fausse tout alors. C'est pas bien.

110. Fabien : Oui, Oui, exactement tu as raison.

111. Nathan: Vous êtes d'accord avec moi, Maître Fabre?

112. Fabien : Je suis entièrement d'accord avec votre proposition qui est satisfaisante.

113. Nathan: Je suis tout à fait content que nous tombions en accord.

114. Céline: Je me réjouis fortement.

115. Nathan : Monsieur Wilfrid quelle est votre opinion SVP ?

116. Max : Là déjà oui.

Lors de désaccords, l'expression personnelle autoritaire d'un élève sur la base de considérations stratégiques peut conduire à un accord dans le groupe comme dans l'extrait suivant.

145. Nathan: Encore une recherche faussée, je trouve.

146. Caroline : Génétiquement modifiée!

147. Céline : Oui.

148. Nathan : On peut directement annuler ces deux expériences car... elles sont faussées, donc...

149. Fabien : Non, non, elles ne le sont pas. Celle-là, elle va nous aider pour nous défendre puisque les souris au bout de vingt heures par jour pendant dix-huit mois : " aucune augmentation dans le taux de tumeurs du sein de ces souris n'a été observée ", donc ça veut dire qu'elles n'ont pas eu d'augmentation de cancer.

150. Céline : Oui, mais tout à l'heure, ce n'était pas la même chose, là c'est le sein, et là, la c'est [inaudible] observée. Donc peut-être que suivant les trucs c'est pas pareil.

151. $[\ldots]$

152. Nathan: De toute façon, ce n'est pas valide, elles sont génétiquement modifiées.

153. Fabien : Oui, ça, ça va nous aider... donc ça sera valide.

À deux autres reprises, une expression personnelle autoritaire de l'élève tenant un rôle de "leader » a lieu afin de conclure un accord sur un objet discuté dans le groupe. On note également qu'à plusieurs reprises des interventions conflictuelles ont lieu. Ceci indique que les relations entre élèves influent sur les objets de discours collectivement élaborés et structurent la discussion.

\section{DISCUSSION}

Différents procédés collaboratifs ont été identifiés dans les groupes de discussion. Les procédés d'élaboration collective des savoirs les plus simples sont par exemple basés sur la répétition ou l'acquiescement: un élève fait une proposition et les autres l'acceptent sans discuter. II est parfois, à cette occasion, fait usage d'autorité. Des procédés plus complexes se manifestent dans des processus collaboratifs, par exemple lorsqu'un élève fait une proposition, reprise ou développée par un autre, et prolongée dans les successions de tours de parole. Ceci peut avoir lieu entre plusieurs membres du groupe de discussion ou en dyades au sein du groupe et à plusieurs occasions, les élèves ont adopté les rôles du jeu dans ces procédés discursifs. Lors de confrontations de points de vue opposés, les élèves peuvent dans certains cas, développer des procédés complexes pour dépasser leurs désaccords et articuler leurs positions opposées. De même, la prise en compte du discours potentiel de l'autre groupe (position opposée) permet dans certains cas le développement de justifications et une contre-argumentation collaborative entre deux élèves. Dans d'autres cas, au contraire, des confrontations peuvent conduire les élèves à abandonner l'objet de discours et provoquer des tensions interpersonnelles.

Les désaccords parviennent à être résolus par la discussion lorsqu'ils portent sur la stratégie, la formulation d'arguments, l'interprétation des textes mais conduisent à une déstabilisation de l'objet de discours lorsqu'ils sont porteurs d'une exigence épistémologique. Des désaccords sont déplacés sur un registre ontologique et résolus par des procédés collaboratifs, d'autres sont résolus sur la base de considérations stratégiques, d'autres enfin ne parviennent pas à être dépassés. Dans ce cas, les élèves peuvent exprimer individuellement leur point de vue lors de votes ou abandonner l'objet de discours. Des considérations stratégiques peuvent alors permettre (mais pas toujours) l'adoption d'une position collective.

Cette étude souligne l'importance des considérations de nature épistémologique et des connaissances profanes des élèves confrontés à une controverse socio-scientifique. L'analyse montre également que les relations entre les élèves influencent leur discussion collaborative. Les désaccords causant des tensions interpersonnelles, les élèves sont parfois amenés à adopter les positions d'avocats du jeu de rôle pour discuter leurs points de vue et cette stratégie 
semble leur fournir un moyen d'éviter d'explorer leurs désaccords.

Nous avons procédé à l'analyse microscopique des échanges langagiers des élèves sans séparer la cognition du social, les connaissances et rôles sociaux étant développés par les élèves dans leurs interactions. Cette analyse a indiqué que les rôles sociaux établis dans les interactions structurent les connaissances élaborées dans les groupes.

Nous avons observé que les processus d'argumentation collaborative sont le plus souvent développés entre deux élèves dans les groupes de discussion. De plus, cette co-construction d'arguments en dyades se produit lorsque les élèves adoptent des rôles sociaux distincts, l'un agissant comme un "leader " et l'autre comme un "aide" qui collabore avec le leader. Lorsque ces élèves expriment des positions opposées ou lorsque la discussion n'est pas menée par le "leader", des confrontations contradictoires apparaissent et ne peuvent pas être dépassées par la discussion. À l'occasion, un élève dans un rôle d'« aide " soulève des critiques et son intervention crée une opportunité d'argumentation collaborative entre l'élève adoptant le rôle de "leader " et un autre élève agissant comme « aide».

Il apparaît que les procédés collaboratifs plus complexes sont mis en œuvre dans le groupe structuré en équipe avec un leader accepté et compréhensif, l'autre groupe ayant des difficultés à réguler ses interactions sur le plan social. De même, Gayford (1992) a montré que les apprentissages individuels et la motivation sont plus importants pour les groupes ayant travaillé en "équipes démocratiques " ou sous une direction qualifiée de "négociatrice " que sous une direction " dominatrice ", "dévolutrice " ou selon les conseils d'observateurs critiques.

Par ailleurs, bien qu'il soit artificiel de séparer interactions sociales et connaissances collectivement élaborées dans les groupes de discussion (Moje \& Shepardson 1998), nous nous centrons ici, à des fins analytiques, sur les rôles sociaux développés par les élèves dans la discussion de groupe afin de questionner différents facteurs pouvant influencer les positions sociales façonnées dans l'interaction.

On remarque que dans les deux groupes de discussion, il n'y a pas de base égalitaire dans la participation à la discussion, certains élèves ayant très peu participé aux échanges (généralement les filles). Le statut académique, estimé par les notes obtenues par les élèves, semble être un facteur d'influence dans les rôles adoptés par les élèves dans leurs discussions de groupe. Ceci est en accord avec les résultats de Richmond \& Striley (1996) : dans le groupe A, les deux élèves se trouvant exclues des discussions sont des élèves dont les notes en sciences physiques sont les plus faibles de la classe; le leader est un élève de niveau élevé. Dans le groupe $B$, l'élève qui peut être décrit comme un leader contesté a obtenu la moyenne la plus faible de la classe.

Si l'on compare avec les moyennes au premier trimestre dans l'ensemble des disciplines, on constate d'autre part que trois filles ont obtenu les trois moyennes les plus élevées, ce qui semble indiquer que ce soient les notes en sciences qui constituent des facteurs d'influence importants dans les positionnements des élèves au cours de cette activité.

De plus, dans la mesure où les pratiques discursives des élèves dépendent de leur contexte de production, il convient de questionner l'impact de la situation pédagogique sur les discours des élèves. Les différences observées dans les procédés d'élaboration collective des savoirs dans les deux groupes de discussion amènent à s'interroger sur l'équité face à l'apprentissage dans ce type d'activités (Kelly et al., 2001). Cela conduit à soulever la question de la stratégie pédagogique des travaux en petits groupes. Kelly et Crawford (1997) suggèrent d'insister sur le rôle médiateur de l'enseignant dans ce contexte d'enseignement particulier. Dans cette étude, l'enseignante intervient à plusieurs reprises pour préciser les consignes de la tâche et veiller à la gestion du temps. Ce faisant, elle interrompt les discussions entre élèves. Par ailleurs, Jiménez-Aleixandre et al. (2000) ont montré l'influence de la culture scolaire sur l'argumentation des élèves sur une question socioscientifique. Comme le souligne Simonneaux (2003, p. 204), "les situations de débat en classe engendrent des difficultés du côté des enseignants qui doivent changer de posture: devenir des gestionnaires de débat au lieu d'être ceux qui savent. " II est alors important de proposer aux enseignants des formations à la gestion pédagogique de discussions sur des questions socio-scientifiques.

Par ailleurs, Dawes (2004) propose de former les élèves à la pratique du langage lors des discussions de groupe afin de favoriser les discours "d'exploration " (Mercer, 1996). Dans la même veine, Grace (2005) considère que des outils de « guidage » fournissant des règles pour les discussions collaboratives seraient utiles aux élèves pour organiser leurs discussions en groupes. Nous adhérons à ces propo- 
sitions et les complétons pour l'étude de questions scientifiques socialement vives. Oulton et al. (2004) ont précisé qu'il est difficile d'organiser un débat effectif en classe en référence à Clarke (1992) pour qui nous vivons dans un temps de déclin généralisé de discours et de civilités. Les discussions doivent alors être informées pour ne pas être seulement des " échanges d'ignorance " (Clarke, 1992). On peut ainsi penser que l'étude menée ici à des fins de recherche pourrait servir en classe un travail d'exploration avec les élèves des procédés discursifs afin d'identifier les contraintes qui interviennent dans l'appréhension de la controverse. Confrontés aux incertitudes de "la science en train de se faire " et défendant des points de vue opposés, les élèves ont par exemple tenté de résoudre leurs désaccords dans des procédures de vote. Cela interroge sur les modalités de prises de décision en contexte d'incertitude et face à des discours scientifiques contradictoires. Driver et al. (1996) ont montré que lors de l'étude de controverses socio-scientifiques, une minorité d'élèves considère qu'obtenir une certitude scientifique est impossible et considèrent pour la plupart que la controverse sera résolue lorsque suffisamment de données empiriques seront disponibles. Dans ce contexte, les élèves ont tendance à compartimenter les données scientifiques et l'information qu'ils utilisent pour prendre une décision personnelle (Zeidler et al., 2002) et appuient leurs décisions sur d'autres considérations en attente de preuves scientifiques (Albe, 2005). Ceci ne nous apparaît pas sans lien avec une certaine conception des sciences ou une "culture de l'évidence " héritée du scientisme et du positivisme qui concourt à déléguer aux experts scientifiques la décision face à une question incertaine et controversée. Afin de favoriser une "culture de l'argumentation" (Breton, 1996), nous considérons que l'étude par les élèves de controverses socio-scientifiques doit s'accompagner de l'examen explicite en classe de la façon dont des faits scientifiques sont produits au sein d'une communauté et en particulier du rôle de la controverse et de l'argumentation dans cette élaboration. Plusieurs auteurs ont souligné le besoin d'enseignements explicites portant sur l'épistémologie et la sociologie des sciences (Bader, 2004 ; Driver et al., 1996 ; Ryder, 2002). Plus particulièrement, considérer les sciences comme des pratiques sociales et des constructions rhétoriques (Osborne, 2001) avec ses incertitudes, ses limites, les intérêts en jeu ainsi que considérer le statut, le rôle et les limites des preuves scientifiques nous semblent très important pour l'étude de questions socio-scientifiques.

\section{CONCLUSION}

Parmi les débats portant sur l'alphabétisation scientifique dans de nombreux pays, l'étude par les élèves de controverses socio-scientifiques a récemment été développée. Afin de contribuer à la compréhension de la façon dont les élèves appréhendent ces controverses, nous avons réalisé en classe une étude sur la question de la dangerosité des téléphones cellulaires avec des élèves de $1^{\text {re }}$ de série technologique de l'enseignement agricole. Dans une approche microethnographique, nous avons analysé les pratiques discursives des élèves en groupes de discussion.

Cette étude souligne comme d'autres travaux (Alexopoulou \& Driver, 1996) les inter-relations complexes entre des facteurs cognitifs, contextuels et sociaux qui influencent l'élaboration de connaissances dans ces situations. Contrairement à des recherches où les discussions entre élèves portent principalement sur des aspects procéduraux des activités (Bianchini, 1997 ; Kittleson \& Southerland, 2004), les discussions portent ici sur l'étude des textes et l'élaboration d'arguments. Les élèves se sont ainsi montré aptes à appréhender cette controverse. Pour Osborne et al. (2004), les questions socioscientifiques offrent la possibilité de développer l'argumentation des élèves. II semble qu'ici la controverse proposée a permis un grand engagement des élèves, ce qui souligne l'intérêt éducatif du traitement de questions socio-scientifiques en classe. Étant une étude de cas, il n'est pas question de généraliser les résultats obtenus, mais soulignons cependant que les analyses conduites tendent à indiquer que l'étude de controverses socio-scientifiques apparaît comme une activité porteuse de grandes exigences sur le plan épistémologique et social.

Virginie Albe virginie.albe@educagri.fr

École nationale de formation agronomique (Toulouse) Unité propre "Didactique des questions scientifiques, économiques et sociales émergentes" 


\section{NOTES}

(1) Voir par exemple le programme du colloque « La crise mondiale des sciences ": Lille, novembre 2005.

(2) La note de service du ministère de l'Agriculture et de la Pêche $\mathrm{n}^{\circ}$ 2000-2072 en date du 18 juillet 2000 (France, 2000) précise que lors de «l'analyse contradictoire de la fiabilité des connaissances (exemples actuels des OGM, effet de serre, etc.) [...] les étudiants poursuivront à cette occasion le développement de leur conscience citoyenne [...] Les débats offriront un champ d'exercice privilégié à cette méthode ».
(3) La leçon peut être consultée sur le site internet de l'École nationale de formation agronomique (ENFA), à l'adresse : http:// www.enfa.fr/cdrv/layout.php?ct=pc/disc-pc.htm (consulté le 7 septembre 2006)

(4) L'ensemble des verbatim peuvent être consultés sur le site internet de l'ENFA, à l'adresse suivante: http://www.enfa.fr/cdrv/ layout.php?ct=pc/disc-pc.htm (consulté le 7 septembre 2006).

\section{BIBLIOGRAPHIE}

AikenHEAD G. (2003). "Review of Research on Humanistic Perspectives in Science Curricula ". Paper Presented at European Science Education Research Association (ESERA) 2003 Conference. Pays-Bas, 19-23 août 2003.

Albe V. \& Simonneaux L. (2003). «Procès sur les téléphones mobiles: impact sur la réflexion épistémologique d'enseignants ". In V. Albe, C. Orange \& L. Simonneaux (éd.), Recherches en didactique des sciences et des techniques: questions en débat. Toulouse: ARDIST \& ENFA, p. 253-260.

Albe V. (2005). «Positions d'étudiants et d'étudiantes sur une question techno-scientifique controversée: la dangerosité des téléphones cellulaires ». Revue canadienne de l'enseignement des sciences, des mathématiques et des technologies, vol. 5, $\mathrm{n}^{\circ} 3$, p. 361-376.

Alexopoulou E. \& Driver R. (1997). "Small Group Discussions in Physics: Peer Interaction Modes in Pairs and Fours ". Journal of Research in Science Teaching, vol. $33, n^{\circ} 10$, p. $1099-1114$.

AMIGUES R. (1988). "Travail en groupe des élèves et changement de conception ", Technologies, idéologies, pratiques, vol. $7, \mathrm{n}^{\circ} 2$, p. 117-135 («L'enseignement des circuits électriques : conceptions des élèves et aides didactiques ", éd. par R. Amigues \& S. Johsua).

BADER B. (2003). "Interprétation d'une controverse scientifique: stratégies argumentatives d'adolescentes et d'adolescents québécois". Revue canadienne de l'enseignement des sciences, des mathématiques et des technologies, vol. 3, $\mathrm{n}^{\circ} 3$, p. 231-250.

BADER B. (2004). « Disqualification de points de vue critiques face aux sciences: procédés discursifs de jeunes du secondaire ". Cahiers du Cirade, n 3, p. 5-23.

BARNES D. \& Todd F. (1977). Communication and Learning in Small Groups. London : Routledge \& Kegan Paul.

BARNES D. (1977). " Talking and Writing in Science Lessons ». Cambridge Journal of Education vol. 7, $\mathrm{n}^{\circ} 3$, p. 138-147.

BIANCHINI J. A. (1997). "Where Knowledge Construction, Equity, and Context Intersect: Student Learning of Science in Small Groups ». Journal of Research in Science Teaching, vol. 43, p. 1039-1065.

BILLIG M. (1987). Arguing and Thinking : A Rhetorical Approach to Social Psychology. Cambridge: Cambridge University Press.

Black P. \& AtKIn M. J. (1996). Changing the Subject, Innovation in Science, Mathematics and Technology Education. London : Routledge ; Paris : OCDE.
BRETON P. (1996). L'argumentation dans la communication. Paris : La Découverte.

Brown J. S. ; Collins A. \& Duguid P. (1989). "Situated Cognition and the Culture of Learning. Educational Researcher, vol. 17, $\mathrm{n}^{\circ} 1$, p. 32-42.

ClARKE P. (1992). "Teaching Controversial Issues». Green Teacher, $\mathrm{n}^{\circ} 31$, p. 29-32.

Coulter J. (1979). The social Construction of Mind : Studies in Ethnomethodology and Linguistic Philosophy. Totowa [NJ] : Rowan \& Littlefield.

DAWES L. (2004). « Talk and Learning in Classroom Science ». International Journal of Science Education, vol. 26, p. 677-695.

Desautels J. ; Larochelle M. ; Gagne B. \& Ruel F. (1993). "La formation à l'enseignement des sciences: le virage épistémologique ». Didaskalia : recherches sur la communication et l'apprentissage des sciences et des techniques, $\mathrm{n}^{\circ} 1$, p. $79-67$

DeWhurst D. W. (1992). "The Teaching Of Controversial Issues ". Journal Of Philosophy of Education, vol. 26, $\mathrm{n}^{\circ} 2$, p. 153-163.

Driver R. ; Leach J. ; Millar R. \& Scott P. (1996). Young People's Image of Science. Buckhingham : Open University Press.

Driver R. ; Newton P. \& Osborne J. (2000). « Establishing the Norms of Scientific Argumentation in Classrooms ". Science Education, vol. 84, n 3, p. 287-312.

EdWARds D. \& Mercer N. (1987). Common Knowledge: The Development of Understanding in the Classroom. New York : Routledge.

EISENBERG N. (2000). "Emotion, Regulation and Moral Development ». Annual Review of Psychology, vol. 51, p. 665-697.

Fillon P. \& Peterfalvi B. (2004). "L'argumentation dans l'apprentissage scientifique au collège ". Aster : recherches en didactique des sciences expérimentales, $\mathrm{n}^{\circ} 38$, p. 151-184.

Fillon P. \& Peterfalvi B. (2005). « Argumentation et différenciation conceptuelle en sciences $"$. In $4^{\text {es }}$ rencontres de I'ARDIST : didactique des sciences et des techniques: contributions: Lyon, 10-15 octobre 2005. Lyon : INRP, p. 151-158.

FLEMING R. (1986). "Adolescent Reasoning in SocioScientific Issues, Part II: Non-Social Cognition". Journal of Research in Science Teaching, vol. $23, \mathrm{n}^{\circ} 8$, p. 689-698. 
FourEZ G. (2002). "Les sciences dans l'enseignement secondaire ". Didaskalia : recherches sur la communication et l'apprentissage des sciences et des techniques, $\mathrm{n}^{\circ} 21$, p. 107-122.

France: MINISTÈre DE L'ÉdUCATION NATIONALE : “L'enseignement des sciences au lycée ". Bulletin officiel de l'Éducation Nationale, n 6, hors-série, 12 Août 1999.

France: Ministère dE L'Agriculture ET DE LA PÊCHE. Programme du baccalauréat S, rentrée 2000. Note de service $\mathrm{n}^{\circ} 2000-2072,18$ juillet 2000.

GarciA-Debanc C. (1995). "Interactions et construction des apprentissages dans le cadre d'une démarche scientifique ". Repères : recherches en didactique du français langue maternelle, $\mathrm{n}^{\circ} 12$, p. 79-103.

GAYFORD C. (1992). "Patterns of Group Behaviour in OpenEnded Problem Solving in Science Classes of 15-YearOld Students in England ». International Journal of Science Education, vol. 14, $\mathrm{n}^{\circ} 1$, p. 41-49.

GILLY M. (1988). "Interactions entre pairs et constructions cognitives: modèles explicatifs". In A.-N. PerretClermont \& M. Nicolet (éd.). Interagir et connaître : enjeux et régulations sociales dans le développement cognitif. Cousset [Confédération Helvétique] : Delval, p. 19-28.

GofFARD M. \& GofFARD S. (2003). «Interactions entre élèves et résolution de problèmes. Aster : recherches en didactique des sciences expérimentales, $\mathrm{n}^{\circ} 37, \mathrm{p} .165-187$.

Goffman E. (1974). Les rites d'interaction. Paris : Éd. de Minuit.

Grace M. M. (2005). « Developing Personal Values and Argumentation Skills Through Decision-Making Discussions About Biological Conservation ». Paper presented at the ESERA Conference: Barcelona, 28 août-1 ${ }^{\text {er }}$ septembre 2005.

GRISON B. (2004). "Des sciences sociales à l'anthropologie cognitive : les généalogies de la cognition située, Activités, vol. $1, \mathrm{n}^{\circ} 2$, p. 26-34. Disponible au format PDF sur Internet à l'adresse : http://www.activites.org/v1n2/ grison.pdf (consulté le 31 mai 2006).

HILLS G. L. C. (1989). “Students' “Untutored” Beliefs About Natural Phenomena: Primitive Science or Commonsense ? "Science Education, vol. 73, n² 2, p. 155-186.

HIND A. : LeACH J. \& RYDER J. (2001). Teaching About the Nature of Scientific Knowledge and Investigation on AS/ A Level Science Courses. Technical Report : University of Leeds (United Kingdom). Disponible sur internet à l'adresse :http://www.nuffieldcurriculumcentre.org/go/ minisite/Post16TeachingAboutScience/Page_22.html

Hind A. ; Leach J. ; Ryder J. \& Prideaux N. (2001). Teaching About the Nature of Scientific Knowledge and Investigation on AS/A Level Science courses. Leeds : CSSME. Document inédit, disponible sur Internet à l'adresse: http://www.education.leeds.ac.uk/research/cssme/Nuff ProjReport.pdf

Hodson D. (2003). «Time for Action : Science Education for An Alternative Future ". International Journal of Science Education, vol. 25, $n^{\circ} 6$, p. 645-670.

JACoBI D. \& Peterfalvi B. (2004). «Interactions langagières : regards didactiques, regards linguistiques". Aster: recherches en didactique des sciences expérimentales, $\mathrm{n}^{\circ} 38$, p. 3-13.
JAUbeRt M. \& ReBIÈre M. (2001). «Pratiques de reformulation et construction de savoirs ". Aster: recherches en didactique des sciences expérimentales, $\mathrm{n}^{\circ} 33, \mathrm{p}$. 81-110.

JiMENEZ-AleiXANDRE M.-P. (2003). «Argumentation et opérations épistémiques au laboratoire ". Communication au symposium Argumentation et apprentissage des sciences, sous la responsabilité d'A. Tiberghien \& Ch. Plantin. Lyon : Institut des sciences de l'homme, 7 novembre 2003.

Jimenez-Aleixandre M.-P. ; Bugallo-Rodriguez A. \& Duschl R. A. (2000). " "Doing the Lesson" or "Doing Science" : Argument in High School Genetics ". Science Education, vol. 84, $n^{\circ} 6$, p. 757-792.

KeLlY G. J. \& CRAWFORD T. (1996). «Student's Interaction with Computer Representations: Analysis of Discourse in Laboratory Groups ». Journal of Research in Science Teaching, vol. 33, $\mathrm{n}^{\circ} 7$, p. 693-707.

KelLy G. J. \& CRAWFORd T. (1997). "An Ethnographic Investigation of the Discourse Processes of School Science ». Science Education, vol. 81, n 5 , p. 533-559.

Kelly G. J. ; Crawford T. \& Green J. (2001). "Common Task and Uncommon Knowledge: Dissenting Voices in the Discursive Construction of Physics Across Small Laboratory Groups ». Linguistics and Education, vol. 12, $\mathrm{n}^{\circ} 2, \mathrm{p} ; 135-174$

KitTLESON J. M. \& SoutherLAND S. A. (2004). «The Role of Discourse in Group Knowledge Construction: A Case Study of Engineering Students ». Journal of Research in Science Teaching, vol. 41, $\mathrm{n}^{\circ}$ 3, p. 267-293.

LAROCHELLE M. (2002). "Rapport au savoir et socialisation à la cité scientifique ». In Actes des $3^{\text {es }}$ journées d'études franco-québécoises: Paris : 17-18 juin. Paris: université Paris 5-René Descartes, p. 58-76.

LARochelle M. \& Desautels J. (2001). « Les enjeux des désaccords entre scientifiques : un aperçu de la construction discursive d'étudiants et étudiantes ". Revue canadienne de l'enseignement des sciences, des mathématiques et des technologies, vol. 1, $\mathrm{n}^{\circ} 1, \mathrm{p} .39-60$.

LEACH J. \& LEWIS J. (2002). «The role of Students' Epistemological Knowledge in the Process of Conceptual Change in Science ". In M. Limón \& L. Mason (éd.), Reconsidering Conceptual Change. Issues in Theory and Practice. Dordrecht : Kluwer, p. 201-216.

LEMKE J. L. (1990). Talking science : Language, Learning, and Values. Norwood [NJ] : Ablex.

Mercer N. (1996). The Guided Construction of Knowledge : Talk Amongst Teachers and Learners. Clevedon: Multilingual Matters.

Millar R. \& Osborne J. F. [éd.] (1998). Beyond 2000 : Science Education for the Future. London : Nuffield Foundation.

Moje E. B. \& Shepardson D. P. (1998). " Social Interactions and Children's Changing Understanding of Electric Circuits : Exploring Unequal Power Relations in "Peer"Learning Groups ». In B. Guzzetti \& C. Hynd (éd.), Perspectives on Conceptual Change. Mahwah [NJ]: L. Erlbaum, p. 225-234.

MondAdA L. (2005). Chercheurs en interaction. Genève : Éd. Le savoir Suisse.

ORgANIZATION FOR ECONOMIC COOPERATION AND DEVELOPMENT (OECD) (1998). Instrument Design: A Framework for Assessing Scientific Literacy. Report of Project Managers Meeting. Paris : OECD. 
Osborne J. (2001). "Promoting Argument in the Science Classroom: a Rhetorical Perspective ". Revue canadienne de l'enseignement des sciences, des mathématiques et des technologies, vol. 1, n 3, p. 271-290.

Osborne J. ; ERduRAn S. \& Simon S. (2004). «Enhancing the Quality of Argumentation in School Science ". Journal of Research in Science Teaching, vol. 41, $\mathrm{n}^{\circ} 10$, p. 994-1020.

Oulton C. ; Dillon J. \& Grace M. (2004). « Reconceptualizing the Teaching of Controversial Issues ». International Journal of Science Education, vol. 26, $\mathrm{n}^{\circ}$ 4, p. 411-424.

PAtronis T ; Potari D. \& SPILIOTOPOULOU V. (1999). " Students' Argumentation in Decision-Making on a Socio-Scientific Issue : Implications for Teaching ". International Journal of Science Education, vol. 21, n 7, p. 745-754.

Plantin C. (1996). L'argumentation. Paris : Éd. du Seuil.

PonteCorvo C. [éd.] (1993). Cognition and Instruction, vol. 11 $\mathrm{n}^{\mathrm{os}} 3$ \& 4 .

RESNICK L. (1988). "Learning in school and out ». Educational Researcher, vol. 16, $n^{\circ}$ 9, p. 13-20.

RICHMOND G. \& StRILEY J. (1996). « Making Meaning in Classrooms : Social Processes in Small-Group Discourse and Scientific Knowledge Building 》. Journal of Research in Science Teaching, vol. 33, $\mathrm{n}^{\circ}$ 8, p. 839-858.

ROYAL SOCIETY [GB] (1985). The Public Understanding Of Science. London: The Royal Society.

RYDER J. (2002). "School Science Education for Citizenship : Strategies for Teaching About the Epistemology of Science". Journal of Curriculum Studies, vol. 34, $\mathrm{n}^{\circ}$ 6, p. 637-658.

Sader T. D. ; Chambers F. W. \& Zeidler D. L. (2004). “Student Conceptualisations of the Nature of Science in Response to a Socioscientific Issue ». International Journal of Science Education, vol. 26, n4, p. 387-409.

SADLER T. D. \& ZeIDLER D. L. (2004). "The Morality of Socioscientific Issues: Construal and Resolution of Genetic Engineering Dilemmas". Science Education, vol. 88, $\mathrm{n}^{\circ} 1$, p. 4-27.

Sader T. D. \& ZeidleR D. L. (2005). "Patterns of Informal Reasoning in the Context of Socioscientific Decision Making ". Journal of Research in Science Teaching, vol. $42, \mathrm{n}^{\circ} 1$, p. 112-138.

SchneebergeR P. \& Ponce C. (2003). «Tirer parti des échanges langagiers entre pairs pour construire des apprentissages en sciences ". Aster : recherches en didactique des sciences expérimentales, $\mathrm{n}^{\circ} 37, \mathrm{p} .53-82$.
Schubauer-Leoni M.-L. \& Perret-Clermont A.-N. (1980). «Interactions sociales et représentations symboliques dans le cadre de problèmes additifs ". Recherches en didactique des mathématiques, vol. 1, $\mathrm{n}^{\circ}$ 3, p. 297-350.

SCHWEIZER D. M. \& KelLY G. J. (2001). "An Investigation of Student Engagement in a Global Warming Debate ». In Actes de la conférence NARST, Saint-Louis, 25-28 March. Saint-Louis : NARST [CD-Rom].

Shamos M. H. (1995). The Myth of Scientific Literacy. New Brunswick [US] : Rutgers University Press.

Simonneaux L. (2001). "Role-Play or Debate to Promote Students' Argumentation and Justification on An Issue in Animal Transgenesis ". International Journal of Science Education, vol. 23, $\mathrm{n}^{\circ}$ 9, p. 903-928.

SimonneauX L. (2003). "Argumentation dans les débats en classe sur une technoscience controversée ". Aster: recherches en didactique des sciences expérimentales, $\mathrm{n}^{\circ} 37$, p. 189-214.

Solomon J. \& AikenHEAD G. [éd.] (1994). STS Education: International Perspectives on Reform. New York : Teachers College Press.

Strauss A. (1992). La trame de la négociation: sociologie qualitative et interactionnisme / textes réunis et présentés par I. Baszanger. Paris : L'Harmattan.

SutTon C. (1995). "Quelques questions sur l'écriture et la science: une vue personnelle d'outre-manche". Repères: recherches en didactique du français langue maternelle, $\mathrm{n}^{\circ} 12$, p. 37-51.

Toulmin S. (1958). The Uses of Argument. Cambridge: Cambridge University Press. [tr. fr. Les usages de l'argumentation. Paris : PUF, 1993]

UNION EUROPÉENNE (1995). Livre blanc sur l'éducation et la formation: enseigner et apprendre: vers la société cognitive [COM(95) 590]. Bruxelles: Commission européenne.

Weisser M. ; MAsclet E. \& Remigny M.-J. (2003). “ Construction de la compréhension par l'argumentation orale en sciences. Expérience menée au cycle III ". Aster: recherches en didactique des sciences expérimentales, $\mathrm{n}^{\circ} 37$, p. 17-52.

YANG F-Y. \& ANDERSON O. R. (2003). "Senior High School Students' Preference and Reasoning Modes About Nuclear Energy Use ». International Journal of Science Education, vol. 25, n², p. 221-244.

Zeidler D. L. ; Walker K. A. ; Ackett W. A. \& Simmons M. L. (2002). "Tangled Up in Views : Beliefs in the Nature of Science and Responses to Socioscientific Dilemmas ". Science Education, vol. 86, n 3, p. 343-367. 